\title{
Efectos de la planta en cojín Oreopolus glacialis (Rubiaceae) sobre la riqueza y diversidad de especies en una comunidad alto-andina de Chile central
}

\author{
Effects of the cushion plant Oreopolus glacialis (Rubiaceae) on species richness and \\ diversity in a high-Andean plant community of central Chile
}

\author{
ERNESTO I. BADANO, MARCO A. MOLINA-MONTENEGRO, CONSTANZA QUIROZ \& LOHENGRIN A. CAVIERES \\ Laboratorio de Biogeografía Ecológica, Departamento de Botánica, Facultad de Ciencias Naturales y \\ Oceanográficas, Universidad de Concepción, Casilla 160-C, Concepción, Chile; \\ e-mail: ebadano@udec.cl
}

\begin{abstract}
RESUMEN
Las plantas en cojín que crecen en ambientes de alta montaña modifican microclimáticamente su entorno, generando microhábitats favorables para el establecimiento de otras especies, actuando como nodrizas. Varios estudios han demostrado que los cojines contienen una mayor riqueza de especies que los espacios abiertos. Sin embargo, hasta ahora, no se ha evaluado el efecto de los cojines sobre otros parámetros comunitarios como la abundancia de individuos, la diversidad y la equitatividad. En este trabajo se analiza el efecto que posee Oreopolus glacialis, una planta en cojín de la cordillera andina, sobre la riqueza, abundancia, diversidad y equitatividad de la comunidad vegetal en un ambiente de alta montaña ubicado a $1.900 \mathrm{~m}$ en Chile central. Se determino la riqueza y abundancia de especies que crecen tanto sobre cojines de $O$. glacialis como en los espacios abiertos entre cojines. Los resultados mostraron que la riqueza de especies y la abundancia de individuos por unidad de área es mayor dentro de los cojines que fuera de ellos. Sin embargo, los valores de diversidad y equitatividad sobre $O$. glacialis fueron menores que fuera de ellos debido a la presencia de algunas especies dominantes. Análisis particulares de la frecuencia y abundancia de cada especie mostraron que no todas las especies son afectadas de la misma manera por la presencia de este cojín, por lo que $O$. glacialis actuaría como nodriza sólo para una parte limitada de los componentes de la comunidad.
\end{abstract}

Palabras clave: facilitación, efecto nodriza, interacciones positivas, cojines, Andes.

\begin{abstract}
Cushion plants growing in high mountain habitats can modify the microclimate within their canopy generating microhabitats more favorable for the recruitment of other plant species, acting as nurse plants. Although several studies have shown that species richness is higher within cushions than outside them, no attempts have been made in order to assess the effect of cushions on other community aspects such as total and individual abundance, diversity and evenness. In this study we analyzed the effect of Oreopolus glacialis -a cushion plant from the South American Andes- on the species richness, total and individual abundance, diversity and evenness of a plant community located in a high mountain environment at 1,900 $\mathrm{m}$ of altitude in central Chile. Species richness and abundance were determined both within and outside $O$. glacialis cushions. Results showed that, per area, species richness and individual abundance were higher within cushions. In contrast, diversity and evenness within $O$. glacialis cushions were lower than outside. This apparent incongruence was caused by some species being highly dominant within cushions. Analyses of the frequency and abundance of each species showed that not all the species were affected in the same way by the cushions, with only four species growing more frequently within cushions. This suggests that nurse effect of $O$. glacialis is a species-specific phenomenon.
\end{abstract}

Key words: facilitation, nurse effect, positive interactions, cushion plants, Andes.

\section{INTRODUCCIÓN}

Los factores limitantes para el establecimiento de plantas en ambientes de alta montaña suelen ser las bajas temperaturas del aire y del suelo, la inestabilidad del sustrato, la elevada evapotranspiración generada por el viento y la corta duración de la estación de crecimiento
(Billings \& Mooney 1968, Billings 1974, Körner 1999). Así, el reclutamiento de nuevos individuos depende, en muchos casos, de la atenuación de tales condiciones por parte de otros organismos (Billings 1974, Bliss 1985).

Las plantas en cojín son formas de vida adaptadas a las condiciones de frío y aridez extremas que presentan los hábitats de alta montaña en 
zonas templadas y subpolares de ambos hemisferios (Armesto et al. 1980, Pysek \& Lyska 1991), siendo particularmente abundantes en el hemisferio sur (Körner 1995). Las especies en cojín se caracterizan por una alta densidad de ramas y hojas, con entrenodos muy cortos que modelan una arquitectura compacta y achaparrada (Gibson \& Kirkpatrick 1985), la cual puede modificar significativamente las condiciones microclimáticas que se generan en su interior (Körner \& DeMoraes 1979, Körner \& Cochrane 1982, Körner 1999). Hagger \& Faggi (1990), por ejemplo, han registrado disminuciones de hasta un $98 \%$ de la velocidad del viento al interior de plantas en cojín, lo que reduce la pérdida de calor por convección y permite que la temperatura del aire en la superficie del cojín sea mayor que la del ambiente externo (Schulze 1982, Körner \& Larcher 1988). Además, la reducción del viento disminuye la pérdida de agua por evapotranspiración, permitiendo mantener una mayor humedad al interior del cojín (Cavieres et al. 1998).

Varios estudios han documentado que al interior de las plantas en cojín la riqueza de especies vegetales tiende a ser mayor que fuera de ellas, sugiriendo que en este tipo de ambientes, donde las condiciones para el establecimiento son limitantes, los cojines constituirían especies nodrizas para las demás especies presentes en la comunidad (Alliende \& Hoffmann 1985, Pysek \& Lyska 1991, Cavieres et al. 1998, Núñez et al. 1999, Molina-Montenegro et al. 2000, Cavieres et al. 2002).

Callaway (1998) ha propuesto que cuando la facilitación por parte de especies nodrizas ocurre mediante una modificación microclimática del ambiente limitante, como en el caso de los cojines, ésta debería afectar a todas las especies de la comunidad más que ser un proceso específico entre pares de especies. Sin embargo, los estudios anteriormente señalados no han ido más allá del análisis de la riqueza de especies como parámetro de diversidad de la comunidad, lo que deja abiertas diversas interrogantes: ¿cómo responde la riqueza de especies ante distintas dimensiones espaciales de la planta nodriza?, ¿qué ocurre con la diversidad si se incluyen términos de abundancia de especies en su medición?, ¿se hallan las especies representadas de la misma manera dentro que fuera de los cojines?, ¿el efecto del proceso de facilitación es generalizado para todas las especies de la comunidad o es especie-específico?

En este estudio se abordan estas interrogantes comparando la riqueza y diversidad de especies que crecen dentro y fuera de cojines de Oreopolus glacialis (Rubiaceae) en Los Andes de Chile cen- tral, esperando que para áreas equivalentes exista una mayor abundancia de especies creciendo al interior de los cojines que fuera de ellos. Adicionalmente se evaluó si el efecto de los cojines actúa directamente sobre la diversidad de la comunidad vegetal y si este efecto es un fenómeno generalizado hacia todas las especies o constituye un proceso de facilitación especie-específico.

\section{MATERIALES Y MÉTODOS}

\section{Sitio de estudio}

El trabajo se llevó a cabo en el valle del paso internacional Pichachén $\left(37^{\circ} 27^{\prime} \mathrm{S}, 71^{\circ} 18^{\prime} \mathrm{O}\right)$, ubicado a $1.900 \mathrm{~m}$ en la cordillera andina de Chile central. Este sitio se halla flanqueado al oeste por dos elevaciones que alcanzan más de $3.000 \mathrm{~m}$ de altitud, el Volcán Antuco y la Sierra Velluda, las cuales ocasionan una marcada sombra de lluvias hacia el valle, haciéndolo más seco y frío que los ambientes que lo rodean (Santibañez \& Uribe 1990).

Las precipitaciones en la zona alcanzan los $2.300 \mathrm{~mm}$ anuales, concentrándose en los meses de invierno y más del $50 \%$ de las mismas ocurren bajo la forma de nieve. La cubierta de nieve se extiende entre los meses de junio hasta noviembre. El régimen térmico se caracteriza por temperaturas que varían entre una máxima promedio de $21^{\circ} \mathrm{C}$ en enero y una mínima promedio de $0,7^{\circ} \mathrm{C}$ en julio (Almeyda \& Saez 1958).

La estructura general del paisaje corresponde a una estepa alto andina donde la comunidad se halla dominada principalmente por el cojín Oreopulus glacialis, arbustos achaparrados de Acaena spp. y especies subarbustivas del género Adesmia. En este ambiente, también son muy comunes las gramíneas de distintas especies y las hierbas anuales.

\section{Especie estudiada}

Oreopolus glacialis (Poepp.) Ricardi (Rubiaceae), habita en terrenos arenosos de Chile y Argentina. En Chile, se distribuye en la Cordillera de Los Andes desde los $33^{\circ}$ hasta los $50^{\circ}$ de latitud Sur, pudiendo encontrarse hasta los $3.000 \mathrm{~m}$ de altitud. Es una hierba perenne, cespitosa, glabra a excepción de las flores. Sus hojas son pecioladas, imbricadas, de 1 a $3 \mathrm{~cm}$ de largo, de láminas enteras, ovado-agudas, coriáceo-carnosas con estípulas enteras. Presenta de dos a seis flores en el ápice de los tallos, con cáliz de tubo corto y piloso, terminado en cinco dientes; corola amari- 
1la, con tubo largo filiforme, luego abierto en cinco lóbulos amarillos. Esta especie florece en el mes de enero, y su fruto es una cápsula dehiscente que se abre por dos valvas.

\section{Muestreo}

En el sitio de estudio se seleccionaron al azar 55 cojines de Oreopolus glacialis. Previamente se confeccionaron aros metálicos de diferentes tamaños $(20,30,40$ y $50 \mathrm{~cm}$ de diámetro). Sobre cada cojín seleccionado de dispuso un aro metálico de tamaño similar al cojín, procediéndose a identificar y contar todos los individuos encontrados dentro del aro. Para obtener información estadísticamente comparable de las especies que crecen fuera de los cojines, por cada muestra al interior de los cojines se tomó una muestra en el ambiente circundante; esto se realizó colocando el mismo aro metálico a una distancia dos veces su diámetro en una dirección al azar desde el centro de cada ejemplar, identificando y contándose los individuos que crecían en el área demarcada por el aro.

\section{Análisis de los datos}

Los números de especies que crecían en cada microhábitat (dentro o fuera de los cojines) se compararon mediante el ajuste de un modelo lineal múltiple a través de un análisis de covarianza (ANCOVA), considerando la posición como la principal variable explicativa. De manera simultánea, y con objeto de comparar la respuesta de la riqueza de especies a la superficie cubierta por los cojines, el área de las muestras fue utilizada como variable de regresión concomitante en el análisis antes mencionado (Sokal \& Rohlf 1995).

Este modelo lineal asume que la riqueza, tanto dentro como fuera de los cojines, responde de igual manera a incrementos del área y sólo evidencia diferencias en el número de especies entre los dos microhábitats. La hipótesis central de este análisis es que, de existir facilitación, la curva de respuesta de la riqueza dentro de Oreopolus glacialis debería ubicarse por encima de la curva para la posición fuera. Esta situación cambiaría si las variables explicativas, el área y la posición de las especies en este caso, interactúan entre ellas (Neter et al. 1996). Por lo tanto, en una segunda instancia se ajustó un modelo múltiple que incluyera la interacción multiplicativa de las dos variables independientes (Neter et al. 1996, Hastie et al. 2001). Para este modelo se hipotetizó que, de existir un efecto interactivo, la curva respuesta de la riqueza dentro de los cojines no solamente sería mayor que la obtenida para la posición fuera, sino que además debería incrementarse de manera más pronunciada.

Para realizar todos los análisis, los valores del área y el número de especies por muestra fueron transformados mediante logaritmo natural, aunque a los valores del número de especies por muestra se le sumó una unidad a fin de poder incluir en el análisis los valores cero (Williams 1995, 1996).

El mismo modelo de ANCOVA fue aplicado a los valores de abundancia total de individuos en las dos posiciones con objeto de analizar cómo era afectada esta componente de la diversidad por la presencia de $O$. glacialis. En este caso, se consideró abundancia total al número de individuos por $\mathrm{dm}^{2}$ de cada muestra, independientemente de la especie a la que pertenecían.

Con los registros de riqueza y abundancia por especie dentro y fuera de $O$. glacialis se calculó el índice de diversidad de Shannon-Weaver (1949) para cada microhábitat. La abundancia de cada especie en las muestras fue estandarizada por el área para evitar posibles errores atribuibles a la distinta superficie de las muestras. El índice fue calculado siguiendo la formulación tradicional:

$H=\sum_{i=1}^{s} p_{i} \cdot \ln \left(p_{i}\right)$, donde $p_{i}$ es la abundancia relativa de la i-esima especie con respecto a la abundancia total de individuos y $S$ es la riqueza total de las muestras. En este caso los valores $p_{i}$ se estimaron como el cuociente entre la abundancia de la iesima especie y la abundancia de individuos de todas las especies. Una vez obtenidos estos valores de diversidad, se estimó el valor de equitatividad $(J)$ para cada microhábitat a fin de analizar la forma en que está representada la diversidad en términos de las abundancia y dominancia de cada especie. Los valores de equitatividad fueron calculados como: $J=H /$ $H_{\text {max }}$, donde $H$ es el valor observado del índice de diversidad de Shannon-Weaver en cada micrositio y $H_{\max }$ es la diversidad máxima esperada $[\ln (S)]$ (Krebs 1989). Para cada valor de diversidad $H$ se estimó su varianza y los índices obtenidos para cada posición fueron comparados mediante la prueba "t" propuesta por Magurran (1988).

Para discriminar si los cojines afectan de manera distinta a las diferentes especies de la comunidad se comparó la frecuencia observada de cada especie creciendo sobre el cojín con una frecuencia generada por azar (c.f., Slade \& Hall 1999). Con la ayuda del software "Resampling Stat" a partir de la frecuencia total de una especie se generaron 1.000 valores al azar de frecuencia tanto al interior como fuera de los cojines, calcu- 
lándose posteriormente la probabilidad con que la frecuencia observada al interior de cojines puede ser generada por azar (Fortin \& Jacquez 2000). Este análisis individualista por pares de especies permitió discernir si existe algún grado de especialización en la asociación de una especie particular con Oreopolus glacialis o si el efecto de este cojín es generalizado para todas las especies de la comunidad (c.f., Callaway 1998). Adicionalmente se realizaron pruebas " $\mathrm{t}$ " en las cuales se compararon las densidades medias al interior y fuera de los cojines para cada especie, lo que permitió discernir si los cojines, además de influir en las frecuencias de ocurrencia de las distintas especies, también poseen efecto sobre la cantidad de individuos de cada especie que se acumulan sobre ellos.

\section{RESULTADOS}

\section{Riqueza}

En total, dentro y fuera de los cojines, se identificaron 18 especies, siete de las cuales fueron encontradas creciendo exclusivamente sobre Oreopolus glacialis, y sólo una creciendo exclu- sivamente fuera (Tabla 1). Las otras 10 especies fueron registradas en ambos micrositios. El cuociente entre el número de especies dentro y fuera de los cojines es 4,2 , lo que implica que al interior de $O$. glacialis se observó cuatro veces más especies que fuera de ellos.

Los resultados del ANCOVA que sólo consideró la posición en la que crecen las especies y el área de las muestras indicó que ambas variables son significativas para explicar las variaciones de riqueza observadas $\left(\mathrm{F}_{2,105}=58,931 ; \mathrm{P}<0,001 ; \mathrm{R}^{2}\right.$ $=0,529)$. Este modelo indicó que además existiría una mayor riqueza de especies creciendo dentro de Oreopolus glacialis que fuera de éste, y que el área tendría un efecto positivo sobre el número de especies tanto dentro como fuera de los cojines (Fig. 1A).

La inclusión del término interactivo en el modelo lineal del ANCOVA mejoró significativamente el poder explicativo de la posición y del área sobre la riqueza de especies $\left(\mathrm{F}_{3,104}=40,042 ; \mathrm{P}<0,001 ; \mathrm{R}^{2}=0,559\right)$. Aquí se observó que dentro de los cojines, además de crecer un mayor número de especies, la riqueza de especies aumenta de manera más rápida que fuera de ellos a medida que el área de las muestras se incrementa (Fig. 1B).

TABLA 1

Frecuencia y abundancia de individuos de cada especie dentro y fuera de cojines de Oreopolus glacialis. Los valores de $\mathrm{P}$ corresponden a pruebas de aleatorización para la comparación de frecuencias y a la prueba t de Student para la comparación de abundancias de cada especie

Frequency of species and abundance of individuals within and outside Oreopolus glacialis cushions. Values of P resulted from randomization tests in the comparison of frequencies, and from Student t-tests in the comparison of abundances

\begin{tabular}{|c|c|c|c|c|c|c|}
\hline \multirow[t]{2}{*}{ Especie } & \multicolumn{2}{|c|}{ Frecuencia de ocurrencia } & \multirow{2}{*}{$\begin{array}{l}\text { Valor P de la } \\
\text { prueba de } \\
\text { aleatorización }\end{array}$} & \multirow{2}{*}{\multicolumn{2}{|c|}{$\begin{array}{c}\text { Densidad promedio } \\
\text { por especie }\end{array}$}} & \multirow{2}{*}{$\begin{array}{c}\text { Valor P de la } \\
\text { prueba t } \\
\text { de Student }\end{array}$} \\
\hline & Dentro & Fuera & & & $\begin{array}{l}\text { cie } \\
\text { Fuera }\end{array}$ & \\
\hline Acaena integerrima & 16 & 2 & $<0,010 *$ & 0,042 & 0,007 & $<0,010 *$ \\
\hline Acaena pinnatifida & 2 & 2 & 0,688 & 0,001 & 0,003 & 0,383 \\
\hline Adesmia papposa & 2 & 0 & 0,264 & 0,005 & 0,000 & 0,156 \\
\hline Adesmia volckmannii & 8 & 3 & 0,125 & 0,030 & 0,005 & $<0,050 *$ \\
\hline Bromus setifolius & 39 & 6 & $<0,010 *$ & 0,208 & 0,015 & $<0,010 *$ \\
\hline Carex aphylla & 0 & 2 & 0,799 & 0,000 & 0,017 & 0,258 \\
\hline Euphorbia collina & 8 & 6 & 0,385 & 0,014 & 0,013 & 0,946 \\
\hline Jaborosa volckmanii & 1 & 0 & 0,505 & 0,007 & 0,000 & 0,258 \\
\hline Mulinum spinosum & 1 & 0 & 0,484 & 0,001 & 0,000 & 0,320 \\
\hline Olsynium frigidum & 1 & 0 & 0,484 & 0,001 & 0,000 & 0,320 \\
\hline Perezia recurvata & 2 & 2 & 0,693 & 0,007 & 0,003 & 0,558 \\
\hline Poa sp. & 1 & 2 & 0,853 & 0,001 & 0,001 & 0,999 \\
\hline Rytidosperma sp. & 4 & 0 & 0,065 & 0,004 & 0,000 & 0,205 \\
\hline Senecio francisci & 4 & 0 & $<0,050 *$ & 0,012 & 0,000 & $<0,050 *$ \\
\hline Senecio neaei & 14 & 4 & $<0,010^{*}$ & 0,066 & 0,007 & $<0,010^{*}$ \\
\hline Sisyrinchium arenarium & 3 & 0 & 0,125 & 0,006 & 0,000 & 0,219 \\
\hline Tarasa humilis & 1 & 3 & 0,944 & 0,004 & 0,004 & 0,999 \\
\hline Viola cotyledon & 1 & 1 & 0,767 & 0,005 & 0,001 & 0,403 \\
\hline
\end{tabular}



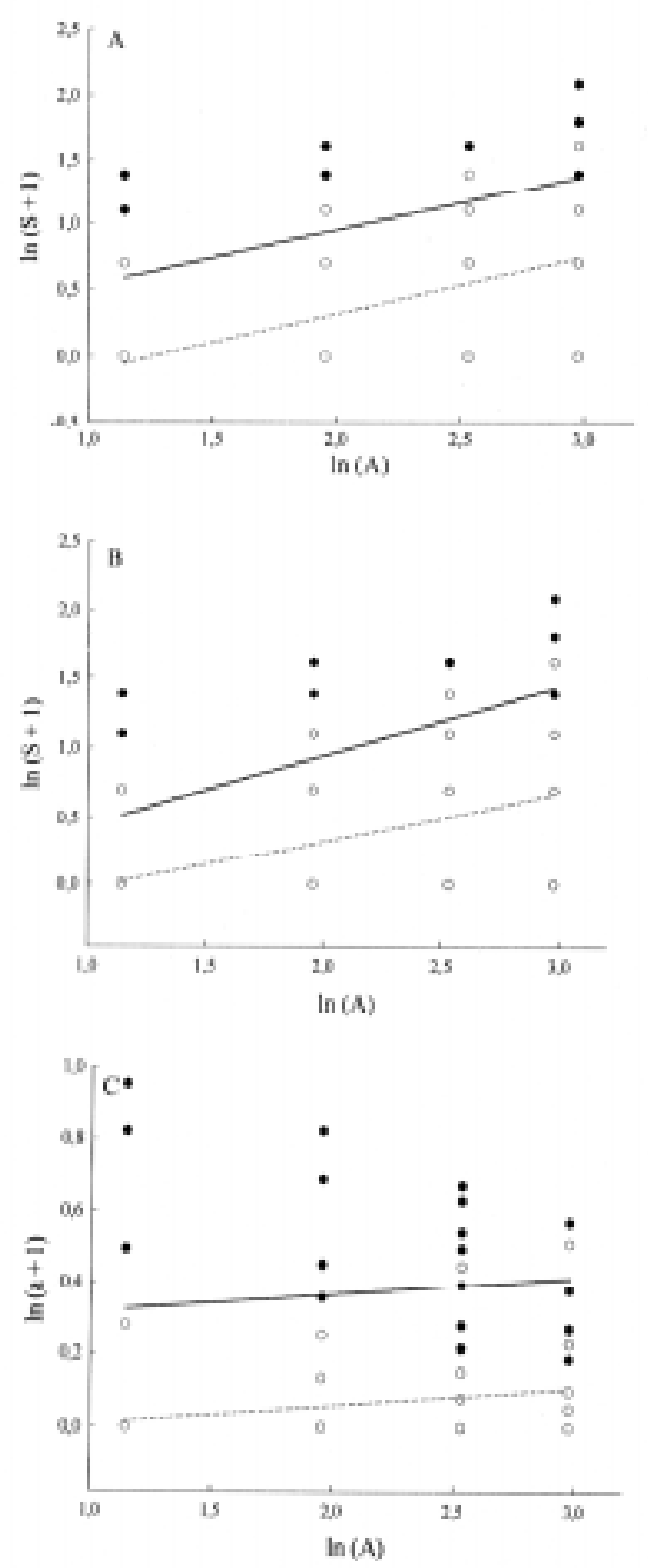

Fig. 1: Variación del número de especies $(S)$ en función del área ( $a$ ) dentro (línea continua) y fuera (línea punteada) de cojines de Oreopolus glacialis. En (A) y (B) se muestra el ajuste de la riqueza para los modelos lineares sin y con interacción entre las variables explicativas, respectivamente. (C) Relaciones ajustadas para la abundancia de individuos $(a)$.

Relationship between number of species $(S)$ and abundance $(a)$ as a function of the sampled area $(A)$ within (solid line) and outside (dashed line) Oreopolus glacialis cushions. (A) and (B) showed the regression lines without and with interactions between factors, respectively. (C) Adjusted regressions for abundance $(a)$.

\section{Abundancias}

La abundancia total de individuos (individuos $\mathrm{dm}^{-2}$ ) mostró ser mayor dentro de los cojines que fuera de ellos y en ambos sitios esta variable se incrementa con el área de la muestra $\left(\mathrm{F}_{2,105}=\right.$ 39,685; $\mathrm{P}<0,001 ; \mathrm{R}^{2}=0,431$ ) (Fig. 1C). Sin embargo, y a diferencia de la riqueza, no se detectó un efecto interactivo de la posición y el área que afectase estas abundancias.

Las especies más abundantes dentro de los cojines fueron Bromus setifolius y Senecio neaei, las que mostraron una clara dominancia en este micrositio (Fig. 2). Fuera de los cojines de Oreopolus glacialis la especie más abundante también fue $B$. setifolius, aunque su abundancia fue muy inferior a la registrada sobre los cojines (Fig. 2). Otra especie importante fuera de los cojines fue Carex aphylla, la cual sólo fue registrada creciendo en este micrositio (Fig. 2).

Pese a que los análisis de regresión mostraron que la riqueza de especies es mayor dentro de los cojines que fuera de ellos, los índices de diversidad de Shannon-Weaver mostraron que al incluir la componente de abundancia por especie, la diversidad fuera de los cojines de Oreopolus glacialis es mayor que dentro de ellos (Fig. 3). La comparación de estos valores reveló que los dos micrositios considerados difieren de manera altamente significativa en lo que refiere a su diversidad $\left(\mathrm{t}_{1,107}=2,465 ; \mathrm{P}=0,007\right)$. Adicionalmente, los índices de equitatividad mostraron que fuera de los cojines las especies se distribuyen de manera mucho más equitativa que dentro de ellos (Fig. 4).

Especificidad de las relaciones con Oreopolus glacialis

Para las frecuencias de ocurrencia de cada especie particular con Oreopolus glacialis, en ninguno de los casos analizados se observó que la frecuencia de ocurrencia disjunta difiriera significativamente de la esperada por azar (Tabla 1). Es decir, ninguna de las especies registradas en este sitio presentaría una preferencia clara por el establecimiento en los espacios abiertos.

Sin embargo, las frecuencias de ocurrencia conjunta con Oreopolus glacialis de Acaena integerrima, Bromus setifolius y las dos especies del género Senecio presentaron diferencias significativas con las frecuencias esperadas de ocurrencia al azar (Tabla 1), indicando que la ocurrencia en estas especies es mayor dentro de los cojines que fuera de ellos. Estas especies, además de presentar una mayor frecuencia de ocurrencia 


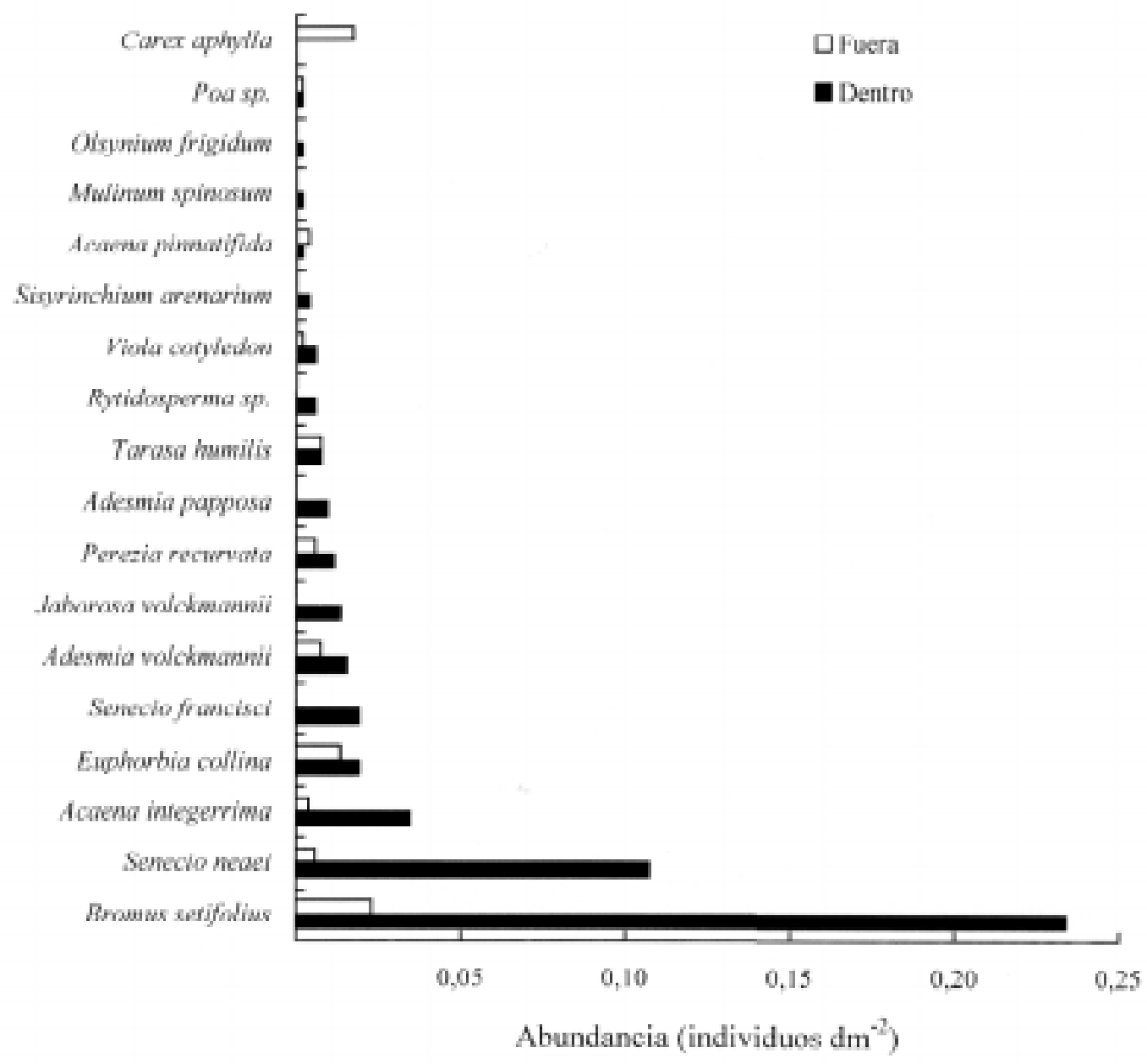

Fig. 2: Abundancia (individuos $\mathrm{dm}^{-2}$ ) de cada especie dentro y fuera de Oreopolus glacialis. Abundance (individuals $\mathrm{dm}^{-2}$ ) of each species within and outside Oreopolus glacialis cushions

dentro de $O$. glacialis, presentaron abundancias de individuos significativamente mayores creciendo en esta posición que fuera de los cojines (Tabla 1). A este conjunto de especies se sumó Adesmia volckmannii, que pese a no presentar una mayor frecuencia de ocurrencia dentro de los cojines, sus abundancias en estos micrositios fueron significativamente superiores que fuera (Tabla 1).

\section{DISCUSIÓN}

El aumento en la riqueza local de especies producido por la presencia de plantas nodrizas es un fenómeno bien conocido y documentado en zonas áridas y semi-áridas (Valiente-Banuet \& Ezcurra 1991, Pugnaire et al. 1996a, Callaway 1997, Callaway \& Pugnaire 1999, Tewksbury \& Lloyd 2001). Sólo en tiempos recientes ha surgido evi-

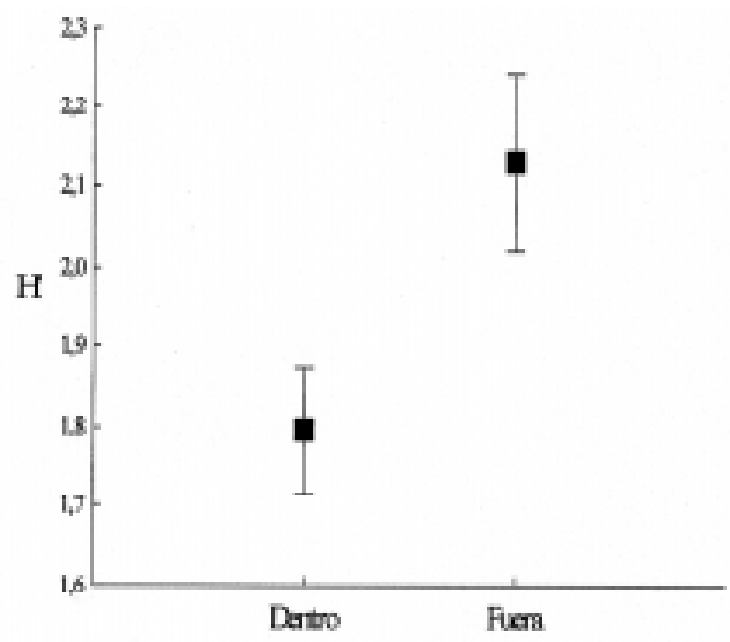

Fig. 3: Valores de los índices de diversidad $(H \pm$ DE) dentro y fuera de cojines de Oreopolus glacialis.

Diversity values $(H \pm \mathrm{SD})$ within and outside Oreopolus glacialis cushions. 


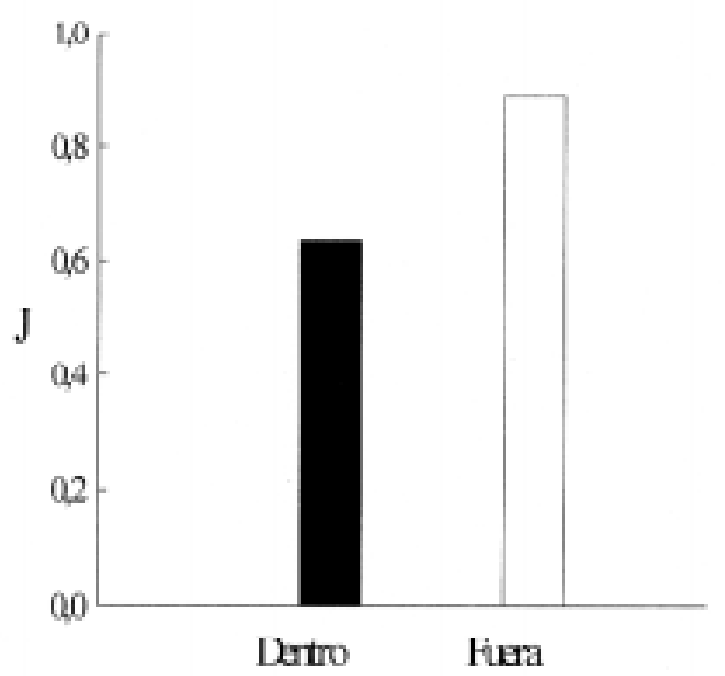

Fig. 4: Valores de equitatividad (J) para la diversidad de especies dentro y fuera de Oreopolus glacialis.

Evenness $(J)$ of the diversity within and outside Oreopolus glacialis cushions.

dencia similar para ambientes de alta montaña (Cavieres et al. 1998, Núñez et al. 1999, MolinaMontenegro et al. 2000, Cavieres et al. 2002, Arroyo et al. en prensa). Por ejemplo, Núñez et al. (1999) encontraron que en el sector andino de la provincia Argentina de Neuquén, la riqueza de especies es mayor en parches dominados por cojines de Mulinum leptacanthum y Oreopolus glacialis. Cavieres et al. (2002) y Arroyo et al. (en prensa) documentan que en la zona andina de la Patagonia Chilena la riqueza de especies es mayor al interior de plantas en cojín que fuera de ellas, especialmente a mayores altitudes, donde el ambiente se torna más limitante para el establecimiento.

En este trabajo, hemos demostrado que dentro de $O$. glacialis no sólo crecen más especies por unidad de área que fuera de ellos, sino que además existe un incremento más rápido de la riqueza dentro de los cojines a medida que aumenta la superficie de los mismos. Este fenómeno, también se reflejó en la abundancia de individuos, sugiriendo que el efecto del tamaño de la nodriza sobre la comunidad vegetal va más allá de la riqueza de especies.

Dos factores podrían estar relacionados con el incremento en el número de especies y la abundancia de individuos con el tamaño del cojín. En primer lugar, nodrizas de mayor área podrían modificar el ambiente con mayor intensidad que aquellas de menor tamaño, ocasionando una atenuación más pronunciada de las condiciones limitantes para el establecimiento de las demás especies de la comunidad. En este aspecto, Pugnaire et al. (1996b) documentaron un incremento en la cantidad de nitrógeno y fósforo conforme se incrementa el área de la nodriza Retama sphaerocarpa (Fabaceae), lo cual proveería a las especies facilitadas "islas de fertilidad" (sensu Pugnaire et al. 1996b) de distinta calidad dependiendo del tamaño de la nodriza. En segundo lugar, se ha propuesto que para plantas en cojín el área es una función directa de su edad (Benedict 1989). Cojines de mayor área se corresponderían con aquellos que llevan más tiempo expuestos a proceso de colonización y, por tanto, la probabilidad de encontrar una especie creciendo sobre ellos es mayor que la de cojines de menor área.

Sin embargo, a pesar de que la riqueza de especies es mayor dentro de los cojines, los índices de diversidad y equitatividad de Shannon-Weaver indicaron que estos atributos son mayores fuera de los cojines. Esta aparente incongruencia podría explicarse por la especificidad encontrada en la asociación al cojín. El hecho de que cuatro de las dieciocho especies encontradas en este estudio (ver Tabla 1) fueran detectadas creciendo de manera más frecuente dentro de Oreopolus glacialis que fuera de ellos, estarían mostrando que la magnitud del efecto positivo de este cojín no sería necesariamente balanceado para todas las especies, existiendo especies que se verían más beneficiadas que otras. Adicionalmente, las pruebas destinadas a evaluar las abundancias particulares de cada especie en las dos posiciones revelaron que las mismas cuatro especies son más abundantes al interior de los cojines que fuera de ellos. Estos resultados sugieren entonces que los ensambles que se generan al interior de los cojines si bien son más ricos en especies, son altamente dominados por unas pocas especies, mientras que los ensambles que se forman fuera son más pobres en especies pero con una mayor equitatividad en relación a la abundancia que alcanza cada especie.

Estudios previos realizados por Cavieres et al. (1998) y Molina-Montenegro et al. (2000) sobre cojines de Laretia acaulis Gill et. Hook (Apiaceae) y Azorella trifurcata Hook (Apiaceae), respectivamente, mostraron que los efectos positivos de estas plantas sobre las demás especies de la comunidad no son equitativos $\mathrm{y}$, por tanto, estos autores han sugerido que tales cojines actuarían como nodrizas sólo para una parte de los componentes de la comunidad mientras que el resto de las especies no se vería afectada de ninguna manera por la presencia de los cojines. Los resultados obtenidos con Oreopolus glacialis permitirían reforzar y ampliar la propuesta de los autores 
anteriormente señalados. El efecto nodriza de las plantas en cojín a lo largo de Los Andes esta dirigido sólo hacia algunas especies y no es un fenómeno generalizado para todos los componentes de la comunidad. En este aspecto, Callaway (1998) ha propuesto que aunque el ambiente entregue micrositios favorables para el establecimiento que no estén asociados a plantas nodrizas, algunas especies de la comunidad podrían presentar una tendencia a establecerse preferentemente junto a nodriza involucrando algún tipo de reconocimiento especie-específico. En el sistema bajo estudio en este trabajo, la tendencia que mostraron algunas especies a establecerse preferentemente dentro de los cojines permite especular acerca de la existencia de algún tipo de reconocimiento con el sustrato donde se establecerán.

Gran parte de las plantas que actúan como nodrizas son consideradas pioneras que permiten la colonización y/o mantenimiento de las poblaciones de otras especies en sistemas perturbados o con elevados niveles de estrés (Armesto \& Pickett 1985, Fuentes et al. 1986, Callaway \& Walker 1997, Levine \& Hacker 1998, Quinos et al. 1998). Aunque las plantas en cojín no siempre son consideradas pioneras en ecosistemas de alta montaña, estas formas de vida indudablemente pertenecen a los primeros estados de la sucesión en ambientes áridos y fríos (Raven 1973, Gibson \& Kirkpatrick 1985, Lough et al. 1987, Pyšek \& Liska 1991). Esto permite suponer que estas formas de vida podrían actuar como facilitadoras para las especies tardías de la comunidad en ambientes que presentan condiciones muy limitantes (humedad, nutrientes, luz, etc.) para el reclutamiento (Núñez et al. 1999). Así, en este tipo de ecosistemas, la probabilidad de encontrar una especie cualquiera asociada a una nodriza sería mucho mayor que la de encontrarla fuera de ella.

\section{AGRADECIMIENTOS}

A Maritza Mihoc por su ayuda en la recolección de datos en terreno y la identificación de especies. A María José Parra por su ayuda en terreno. A Chris Lusk por facilitarnos el uso del software "Resampling Stat". Esta es una contribución del "Grupo de Investigación Avanzada en Biología Vegetal", Dirección de Investigación-Universidad de Concepción 201.111.025-1.4. y P99-103 F ICM - Center for Advanced Studies in Ecology and Research in Biodiversity.

\section{LITERATURA CITADA}

ALLIENDE MC \& AJ HOFFMANN (1985) Plants intruding Laretia acaulis (Umbelliferae), a high Andean cushion plant. Vegetatio 60: 151-156.

ALMEYDA E \& F SAEZ (1958) Recopilación de datos climáticos de Chile. Proyecto 14, Investigaciones Económicas Agrícolas, Ministerio de Agricultura, Dirección General de Producción Agraria y Pesquera, Departamento Técnico Interamericano de Cooperación Agrícola, Santiago, Chile. 183 pp.

ARMESTO JJ, MTK ARROYO \& C VILLAGRÁN (1980) Altitudinal distribution, cover and size structure of umbelliferous cushion plants in the high Andes of Central Chile. Acta Oecologica, Oecologia Generalis 1: 327-332.

ARMESTO JJ \& STA PICKETT (1985) A mechanistic approach to the study of succession in the Chilean matorral. Revista Chilena de Historia Natural 58: 917.

ARROYO MTK, LA CAVIERES, A PEÑALOZA \& MA ARROYO-KALIN (en prensa) Positive associations between the cushion plant Azorella monantha (Apiaceae) and alpine plant species in the Chilean Patagonian Andes. Plant Ecology.

BENEDICT JB (1989) Use of Silene acaulis for dating: the relationships of cushion diameter to age. Arctic and Alpine Research 21: 91-96.

BILLINGS WD (1974) Adaptations and origins of alpine plants. Arctic and Alpine Research 6: 129-142.

BILLINGS WD \& H MOONEY (1968) The ecology of arctic and alpine plants. Biological Reviews 43: 481520.

BLISS LC (1985) Alpine. En: Billings WD \& HA Mooney (eds) Physiological ecology of North American plant terrestrial communities: 41-65. Chapman \& Hall, New York, New York.

CALLAWAY RM (1997) Positive interactions in plant communities and the individualistic-continuum concept. Oecologia 112: 143-149.

CALLAWAY RM (1998) Are positive interactions speciesspecific? Oikos 82: 202-207.

CALLAWAY RM \& L WALKER (1997) Competition and facilitation: a synthetic approach to interactions in plant communities. Ecology 78: 1958-1965.

CALLAWAY RM \& FI PUGNAIRE (1999) Facilitation in plant communities. En: Pugnaire FI \& F Valladares (eds) Handbook of functional plant ecology: 623248. Marcel Dekker, New York, New York.

CAVIERES LA, A PEÑALOZA A, C PAPIC \& M TAMBUTTI (1998) Efecto nodriza del cojín Laretia acaulis (Umbelliferae) en la zona alto-andina de Chile central. Revista Chilena de Historia Natural 71: 337-347.

CAVIERES LA, MTK ARROYO, MA MOLINAMONTENEGRO, C TORRES \& A PEÑALOZA (2002) Nurse effect of Bolax gummigera (Apiaceae) cushion plants in the alpine vegetation of the Chilean Patagonian Andes. Journal of Vegetation Science 13: 547-554.

FORTÍN M \& G JACQUEZ (2000) Randomization test and spatially autocorrelated data. Bulletin of the Ecological Society of America 81: 201-205. 
FUENTES E, A HOFFMANN, A POIANI \& M ALLIENDE (1986) Vegetation change in large clearings: patterns in the Chilean matorral. Oecologia 68: 358-366.

GIBSON N \& JB KIRKPATRICK (1985) A comparison of the cushion plant communities of New Zealand and Tasmania. New Zealand Journal of Botany 23: 549566.

HAGER J \& AM FAGGI (1990) Observaciones sobre distribución y microclima de cojines enanos de la isla de Creta y del noroeste de la Patagonia. Parodiana 6: 109-127.

HASTIE T, R TIBSHIRANI \& J FRIEDMAN (2001) The elements of statistical learning. Springer-Verlag, New York, New York. 533 pp.

KÖRNER C (1995) Alpine plant diversity: a global survey and functional interpretations. En: Chapin ST III \& C Körner (eds) Arctic and alpine biodiversity: 45-62. Springer-Verlag, Berlin, Germany.

KÖRNER CH (1999) Alpine plant life. Springer-Verlag, Berlin, Germany. 338 pp.

KÖRNER CH \& J De MORAES (1979) Water potential and diffusion resistance in alpine cushion plants on clear summer days. Oecologia Plantarum 14: 109120.

KÖRNER CH \& P COCHRANE (1982) Influence of plant physiognomy on leaf temperature on clear midsummer days in the Snowy Mountains, south-eastern Australia. Acta Oecologia, Oecologia Plantarum 4: 117124.

KÖRNER CH \& W LARCHER (1988) Plant life in cold climates. En: Long SF \& FI Woodward (eds) Plants and temperature: 25-57. Cambridge University Press, Cambridge, United Kingdom.

KREBS CJ (1989) Ecological methodology. Harper \& Row, New York, New York. 654 pp.

LEVINE J \& S HACKER (1998) Nitrogen effects on an interaction chain in a salt marsh community. Oecologia 117: 266-272.

LOUGH TJ, JB WILSON, AF MARK \& AC EVANS (1987) Succession in a New Zealand alpine cushion community: a Markovian model. Vegetatio 71: 129138.

MAGURRAN AE (1988) Ecological Diversity and its Measurement. Princeton University Press, Princeton, New Jersey. 179 pp.

MOLINA-MONTENEGRO MA, C TORRES, MJ PARRA \& LA CAVIERES (2000) Asociación de especies al cojín Azorella trifurcata (Gaertn.) Hook. (Apiaceae) en la zona andina de Chile central $\left(37^{\circ} \mathrm{S}\right)$. Gayana Botánica (Chile) 57: 161-168.

NETER J, MH KUTNER, CJ NACHTSHEIM \& W WASSERMAN (1996) Applied linear statistical models. McGraw-Hill, Boston, Massachusetts. 1408 pp.
NÚÑEZ C, M AIZEN \& C EZCURRA (1999) Species associations and nurse plant effect in patches of highAndean vegetation. Journal of Vegetation Science 10: 357-364.

PUGNAIRE FI, P HAASE \& J PUIGDEFABREGAS (1996a) Facilitation between higher plant species in a semiarid environment. Ecology 77: 1420-1426.

PUGNAIRE FI, P HAASE, J PUIGDEFABREGAS, M CUETO, SC CLARK \& LD INCOLL (1996b) Facilitation and succession under the canopy of a leguminous shrub, Retama sphaerocarpa, in a semiarid environment in south-east Spain. Oikos 76: 455464.

PYŠEK P \& L LYSKA (1991) Colonization of Sibbaldia tetrandra cushions on Alpine scree in the Palmiro-Alai Mountains, central Asia. Arctic and Alpine Research 23: 263-272.

QUINOS P, P INSAUTI \& A SORIANO (1998) Facilitative effect of Lotus tenuis on Paspalum dilatatum in a lowland grassland of Argentina. Oecologia 114: 427431.

RAVEN PH (1973) Evolution of subalpine and alpine plant groups in New Zealand. New Zealand Journal of Botany 11: 177-200.

SANTIBÁÑNEZ F \& JM URIBE (1990) Atlas agroclimático de Chile. Regiones VIII y IX. Ediciones Universidad de Chile, Santiago, Chile. 99 pp.

SCHULZE ED (1982) Plant life forms and their carbon, water and nutrition relations. En: Lange OL, PS Nobel, CB Osmond \& H Ziegler (eds) Encyclopedia of plant physiology, Volumen 12B: physiological plant ecology II: 615-676. Springer-Verlag, Berlin, Germany.

SHANNON CE \& W WEAVER (1949) The mathematical theory of communication. University of Illinois, Urbana, Illinois. $144 \mathrm{pp}$.

SLADE N \& D HALL (1999) Randomization test using standard statistical software. Bulletin of the Ecological Society of America 80: 179-180.

SOKAL R \& FJ ROHLF (1995) Biometry: the principles and practice of statistics in biological research. Freeman Company, New York, New York. 887 pp.

TEWKSBURY JJ \& JD LLOYD (2001) Positive interactions under nurse-plants: spatial scale, stress gradient and benefactor size. Oecologia 127: 425424.

VALIENTE-BANUET A \& E EZCURRA (1991) Shade as a cause of the association between the cactus Neobuxbaumia tetetzo and the nurse plant Mimosa luisana in the Tehuacan valley, México. Journal of Ecology 79: 961-971.

WILLIAMS MR (1995) An extreme-value function model of the species incidence and species-area relations. Ecology 76: 2607-2616.

WILLIAMS MR (1996) Species-area curves, the need to include zeroes. Global Ecology and Biogeography Letters 5: 91-93.

Editor Asociado: J. Gutiérrez

Recibido el 7 de mayo de 2002; aceptado el 10 de septiembre de 2002 